\title{
ALGEBRAIC STRUCTURE AND CONFORMAL MAPPING ${ }^{(1)}$
}

\author{
BY \\ MAURICE HEINS
}

1. Given a Riemann surface $F$, let $\mathfrak{A}(F)$ and $\mathfrak{M}(F)$ denote respectively the ring of analytic functions with domain $F$ and the field of meromorphic functions with domain $F$. The following elegant result is due to L. Bers:

If $F$ and $G$ are plane regions and $\phi$ is an isomorphism of $\mathfrak{A}(G)$ onto $\mathfrak{A}(F)$, then there exists either a univalent conformal map $\psi_{1}$ of $F$ onto $G$ or a univalent anticonformal map $\psi_{2}$ of $F$ onto $G$ such that $\phi(g)=g \circ \psi_{1}(g \in \mathfrak{A}(G))$ or $\phi(g)$ $=\bar{g} \circ \psi_{2}(g \in \mathfrak{A}(G))$.

Anterior results of similar character pertaining to rings of bounded analytic functions are due to Chevalley and Kakutani [3]. Rudin [5] has announced the theorem: If $F$ and $G$ are noncompact Riemann surfaces such that there exists an isomorphism between $\mathfrak{A}(F)$ and $\mathfrak{A}(G)$ preserving the complex constants, then $F$ and $G$ are conformally equivalent. The demonstration of Rudin makes use of the existence of an analytic function with assigned zeros on a noncompact Riemann surface (H. Florack [2]).

The primordial concern of the results just cited is conformal equivalence envisaged as a consequence of algebraic equivalence of associated algebraic structures. Now it is immediate that, if one considers a conformal map $\psi$ (not necessarily univalent onto) of a Riemann surface $F$ into a Riemann surface $G$, then $\phi_{\psi}: g \rightarrow g \circ \psi$ defines an isomorphism of $\mathfrak{M}(G)$ into $\mathfrak{M}(F)$ and of $\mathfrak{A}(G)$ into $\mathfrak{A}(F)$. In fact $\phi_{\psi}$ preserves the complex constants and is continuous. It is therefore natural to inquire what can be said when $\mathfrak{M}(G)$ is algebraically equivalent to a subfield of $\mathfrak{M}(F)$ or $\mathfrak{A}(G)$ is algebraically equivalent to a subring of $\mathfrak{A}(F)$ (or more generally $\mathfrak{A}(G)$ admits a homomorphism into $\mathfrak{A}(F)$ ). Stated with this degree of generality the problem is as yet unsolved. Recently, Royden [4] has extended the result of Rudin cited above and has shown using related analytic and algebraic apparatus the following theorem: If $\phi$ is $a$ homomorphism of $\mathfrak{A}(G)$ into $\mathfrak{A}(F)$ which preserves the complex constants, then $\phi(g)=g \circ \psi(g \in \mathfrak{A}(G))$ where $\psi$ is either a conformal map or a constant map of $F$ into $G$. It is assumed that $F$ and $G$ are not compact.

It is to be observed that if $F$ and $G$ are both compact, the result is trivial but the unicity of $\psi$ is lost. If $F$ is compact and $G$ is not compact, the result persists with $\psi$ ùnique and constant.

In the present paper we shall be concerned with isomorphisms of $\mathfrak{M}(G)$ into $\mathfrak{M}(F)$ which preserve the complex constants. Whether Royden's theorem

Received by the editors February 8, 1957.

(1) This research was supported by the United States Air Force through the Air Office of Scientific Research of the Air Research and Development Command. 
has its counterpart for such field isomorphisms remains unsettled. However we shall see that, if a supplementary condition is added then the representation of admitted isomorphisms with the aid of conformal mappings persists. The theorem is

Theorem A. Let $\phi$ denote an isomorphism of $\mathfrak{M}(G)$ into $\mathfrak{M}(F)$ which preserves the complex constants, then

$$
\psi=\{(p, q) \mid(p, q) \in F \times G ; \phi(g)(p)=g(q), g \in \mathfrak{M}(G)\}
$$

is either empty or else is a conformal map of $F$ into $G$.

This result affords a reduction of the general problem for it suffices to be assured that $\psi$ is not empty. It is clear that if $\phi$ do exist which are not realizable with the mediation of conformal maps of $F$ into $G$, then they are quite complicated in character. We shall see further that the following theorem holds.

THEOREM B. If $\phi$ is continuous, then $\psi$ is not empty and hence is a conformal map of $F$ into $G$.

As in the case of the theorems of Rudin and Royden, the analytic apparatus furnished by the results of $\mathrm{H}$. Florack will play an essential role in the proofs of Theorems A and B.

If one is concerned primarily with the question of the interrelation between conformal mappings $\psi$ of a surface $F$ and internal properties of the image subfield induced by $\psi$, then Theorems $\mathrm{A}$ and $\mathrm{B}$ are effective instruments. We shall consider such questions in $\$ \$ 12-16$.

Let $K$ denote a subfield of $\mathfrak{M}(F)$. For each $p \in F$, we define $h_{p}$ as the map $f \rightarrow f(p), f \in K$. The subfield $K$ is said to separate $F$ provided that $p \rightarrow h_{p}$ is univalent. We shall see that the following theorem holds.

THEOREM C. If $K_{1}$ is an arbitrary subfield of $\mathfrak{M}(F)$ containing the complex constants and functions other than constants, then there exist a conformal map $\psi$ of $F$ onto a Riemann surface $G$ and a separating subfield $K_{2}$ of $\mathfrak{M}(G)$ such that $g \rightarrow g \circ \psi$ maps $K_{2}$ onto $K_{1}$. The representation of $K_{1}$ so given is determined up to a conformal equivalence.

2. We turn to the proof of Theorem $C$. The existence part will be treated constructively. Let $G=\left\{h_{p} \mid p \in F\right\}$ where $h_{p}$ is the map $f \rightarrow f(p), f \in K_{1}$. We wish to endow $G$ suitably with a Riemann surface structure. Let $n(p ; f)$ denote the multiplicity at $p$ of a function $f$ meromorphic at $p$. Let $m$ $=\min _{f \in K_{1}} n(p ; f)$ and let $f_{0}$ denote a member of $K_{1}$ such that $m=n\left(p ; f_{0}\right)$. We may assume without loss of generality that $f_{0}(p)=0$. Let $\tau$ denote a local uniformizer for $F$ having as its domain a disk $|z|<\rho^{1 / m}$ and satisfying: $\tau(0)=p, f_{0} \circ \tau=z^{m}$. It follows from the fact that $K_{1}$ is a field containing the complex constants that in the neighborhood of $p$ which is the image of 
$|z|<\rho^{1 / m}$ with respect to $\tau$, each $f$ in $K_{1}$ admits a representation of the form $g_{f} \circ f_{0}$ where $g_{f}$ is meromorphic in $|z|<\rho$.

Suppose $p_{1}$ and $p_{2}$ are distinct points of $F$ for which $h_{p_{1}}=h_{p_{\mathrm{g}}}$. Let $m_{1}$ $=\min _{f \in K_{1}} n\left(p_{1} ; f\right)$ and let $f_{0}$ now denote a member of $K_{1}$ having a zero of order $m_{1}$ at $p_{1}$ and let $m_{2}$ denote the multiplicity of $f_{0}$ at $p_{2}$. Let $\tau_{1}$ and $\tau_{2}$ denote local uniformizers of neighborhoods of $p_{1}$ and $p_{2}$ respectively having as their respective domains $|z|<\rho^{1 / m k}$ and satisfying: $\tau_{k}(0)=p_{k}, f_{0} \circ \tau_{k}$ $=z^{m k}, k=1,2$. Again as a consequence of the fact that $K_{1}$ is a field containing the complex constants we see that for each $f \in K_{1}$ there exists a function $g_{f}$ meromorphic in $|z|<\rho$ such that $f=g_{f} \circ f_{0}$ in the image sets of $\tau_{1}$ and $\tau_{2}$. It is immediate that $m_{2}=\min _{f \in K_{1}} n\left(p_{2} ; f\right)$.

Let $\mathfrak{O}$ denote the family of subsets $O$ of $G$ having the property that if $h_{q} \in O$, then $h_{p} \in O$ for all $p$ in some neighborhood of $q$. It follows that $\mathfrak{D}$ defines a topology on $G$. Let $\psi$ denote the map $p \rightarrow h_{p}$. It carries open sets into open sets. The topology so defined satisfies the Hausdorff separation axiom. Now $\psi$ is continuous. Hence $G$ is connected.

Given $p \in F$, we introduce a local uniformizer $\tau$ as above with domain $|z|<\rho^{1 / m}$ and thereupon define $\sigma_{\tau}$ as the set $\left\{\left(z^{m}, h_{\tau(s)}\right)|| z \mid<\rho^{1 / m}\right\}$. It is a straightforward matter to verify that the family of $\sigma_{\tau}$ endows $G$ with a Riemann surface structure.

With this definition of conformal structure, the map $\psi$ is a conformal map of $F$ onto $G$. We are now in a position to complete the existence part of Theorem C. Given $f \in K_{1}$, let $\tilde{f}=\left\{\left(h_{p}, f(p)\right) \mid p \in F\right\}$. It is readily verified that $\tilde{f}$ is a meromorphic function with domain $G$, and that $K_{2}$, the set of $\tilde{f}$, is a field containing the complex constants. We have $f=\tilde{f} \circ \psi$. Finally we note that $K_{2}$ separates $G$. In fact, if $h_{p} \neq h_{q}$, then for some $f \in K_{1}$, say $f_{0}$, we have $f_{0}(p) \neq f_{0}(q)$ and hence $\tilde{f}_{0}\left(h_{p}\right) \neq \tilde{f}_{0}\left(h_{q}\right)$.

3. It follows from the argument of $\S 2$ that, if $K_{1}$ separates $F$, then for each $p \in F, \min _{f \in K_{1}} n(p ; f)=1$. This need not be the case for an arbitrary subfield $K$ of $\mathfrak{M}(F)$ which separates $F$. Indeed the field $K$ generated by $f(z)$ $\equiv e+z^{2}, g(z) \equiv z^{3}, z \in$ extended plane, separates the extended plane, but $\min _{f \in K} n(0 ; f)=2$.

4. We now consider a conformal map $\sigma$ of $F$ into a Riemann surface $S$ and suppose that $\sigma$ induces an isomorphism of a separating subfield $K_{3}$ of $\mathfrak{M}(S)$ onto $K_{1}: s \rightarrow s \circ \sigma, s \in K_{3}$. We shall show that $\sigma$ admits a representation of the form $\theta \circ \psi$ where $\theta$ is a univalent conformal map of $G$ into $S$. The representation is obviously unique. We note that $s \circ \sigma=g_{s} \circ \psi, g_{s} \in K_{2}$ and that $\{(\psi(p), \sigma(p)) \mid p \in F\}$ is a function. This latter observation follows on remarking that $\psi(p)=\psi(q)$ implies $s(\sigma(p))=s(\sigma(q))$ for all $s \in K_{3}$ and that $K_{3}$ separates $S$. We denote this function by $\theta$. Clearly $\theta$ is continuous. Since $\sigma(p)$ $=\sigma(q)$ implies $g_{8}(\psi(p))=g_{8}(\psi(q))$ and $K_{2}$ separates $G, \theta$ is univalent. We have $\sigma=\theta \circ \psi$ and conclude that $\theta$ is a conformal map.

This result implies that the representation of Theorem $C$ is determined 
up to a conformal equivalence. For suppose that $\sigma$ maps $F$ onto $S$. Then $\theta$ maps $G$ onto $S$. Theorem $C$ is established.

5. We consider some consequences of Theorem C. Given nonconstant meromorphic functions $f, g$ on $F$, there exist a unique analytische Gebilde (B) and a unique conformal map $\phi$ of $F$ into $B$ such that $f=c \circ \phi$ and $g=v \circ \phi$ where $c$ is the map which carries each element of $B$ into its center and $v$ is the map which carries each element of $B$ into its value. A classical realization problem may be stated as follows: Given $f$ meromorphic and nonconstant on $F$, does there exist a (nonconstant) meromorphic function $g$ on $F$ such that the map $\phi$ associated with the ordered pair $(f, g)$ is a univalent map of $F$ onto $\&$ ? ? An ordered pair $(f, g)$ will be termed extendible provided that there exist a conformal map $\psi$ of $F$ into a Riemann surface $H$ which is not a univalent map of $F$ onto $H$ and functions $\tilde{f}, \tilde{g}$ meromorphic on $H$ such that $f=\tilde{f} \circ \psi, g=\tilde{g} \circ \psi$. It may be shown that the $\phi$ induced by an ordered pair $(f, g)$ is a univalent map of $F$ onto (s) if and only if $(f, g)$ is nonextendible. The realization problem is equivalent to determining for a given $f$ a $g$ such that $(f, g)$ is nonextendible.

It is immediate from Theorem $\mathrm{C}$ that, if $(f, g)$ is nonextendible and if $K\{f, g\}$ denotes the subfield of $\mathfrak{M}(F)$ generated by $f, g$ and the complex constants, then $K\{f, g\}$ separates $F$.

We observe that, if $F$ is compact and $K$ is a subfield of $\mathfrak{M}(F)$ which contains the complex constants and separates $F$, then $K=\mathfrak{M}(F)$. In fact, let $f$ denote a nonconstant member of $K$ and suppose that $f$ has valence $n$. If $n=1, F$ is conformally equivalent to the extended plane and $K$ contains all functions of the form $r \circ f$ where $r$ is rational and it follows that $K=\mathfrak{M}(F)$. We put aside the case where the valence of $f$ is 1 . Let $w$ denote a point of the plane over which $f$ is not ramified and let $p_{1}, \cdots, p_{n}$ denote the antecedents of $w$ with respect to $f$. Since $K$ separates, we conclude that there exists $g \in K$ such that $g\left(p_{k}\right)=k, k=1, \cdots, n$. It follows that $(f, g)$ is not extendible and consequently since $F$ is compact that $K\{f, g\}=\mathfrak{M}(F)$.

It follows from this observation that if $G$ of Theorem $C$ is compact, and this is certainly the case for compact $F$, then $K_{1}$ is isomorphic to the field of meromorphic functions on a compact Riemann surface.

6. Proof of Theorem A. Suppose that $\psi \neq \varnothing$. At all events $\psi$ is a function. For if $\left(p, q_{1}\right)$ and $\left(p, q_{2}\right) \in \psi$, we have $g\left(q_{1}\right)=g\left(q_{2}\right), g \in \mathfrak{M}(G)$ and hence $q_{1}=q_{2}$. We first show that the domain of $\psi$ is both open and closed. It follows that the domain of $\psi$ is $F$.

Open. Suppose that $\left(p_{0}, q_{0}\right) \in$ domain of $\psi$. There exists $g_{0} \in \mathfrak{M}(G)$ such that $n\left(q_{0} ; g_{0}\right)=1$. We assume, as we may, that $g_{0}\left(q_{0}\right)=0$. Let $f_{0}=\phi\left(g_{0}\right)$. Let $\Delta$ denote a fixed disk containing $q_{0}$ which is mapped univalently onto a disk $|z|<r$ by $g_{0}$. For each $g \in \mathfrak{M}(G), g$ admits in $\Delta$ a representation of the form $\mathfrak{m}_{g} \circ g_{0}$ where $\mathfrak{m}_{g}$ is meromorphic in $|z|<r$. It follows from the fact that $\phi$ is an isomorphism preserving the complex constants that in some fixed disk 
containing $p_{0} \phi(g)=\mathfrak{m}_{o} \circ f_{0}, g \in \mathfrak{M}(G)$. We conclude that the domain of $\psi$ is open.

Closed. Suppose that $p_{0} \in F$ adhered to the domain of $\psi$ but did not belong to it. There would exist a sequence of points $\left\{p_{k}\right\}_{1}^{\infty}, p_{k} \in$ domain of $\psi$, tending to $p_{0}$. If $\left\{\psi\left(p_{k}\right)\right\}$ were not relatively compact, there would exist a member $g$ of $\mathfrak{A}(G)$ not identically zero which vanished on an infinite subset of the $\psi\left(p_{k}\right)$ [2]. But then $\phi(g)$ would vanish identically, and this is impossible. We conclude that there exists a subsequence of $\left\{\psi\left(p_{k}\right)\right\}$ tending to a point $q_{0} \in G_{0}$. We have $\left(p_{0}, q_{0}\right) \in \psi$ and the assertion follows.

Continuity of $\psi$. If $\psi$ were not continuous at a point $p_{0} \in F$, there would exist a sequence $\left\{p_{k}\right\}$ of points of $F$ tending to $p_{0}$ such that $\psi\left(p_{k}\right) \rightarrow q \neq \psi\left(p_{0}\right)$. But $\left(p_{0}, q\right) \in \psi$ and the contradiction is manifest. The conformality of $\psi$ is now immediate. It is clear that there is a unique map $\psi$ of $F$ into $G$ such that $\phi(g)=g \circ \psi, g \in \mathfrak{M}(G)$.

Corollary. If $\phi$ maps $\mathfrak{M}(G)$ onto $\mathfrak{M}(F)$ and $\psi$ is not empty, then $\psi$ is a univalent map of $F$ onto $G$.

This corollary will be of service in applications.

7. If the Riemann surface $G$ of Theorem A is compact, then $\psi$ is indeed a conformal map of $F$ into $G$. No continuity requirements are imposed on $\phi$.

Genus of $G$ zero. In this case we may take $G$ to be the extended plane. If $f_{0}$ denotes the image with respect to $\phi$ of $g_{0}(z) \equiv z$, then $\phi(g)=g \circ f_{0}, g \in \mathfrak{M}(G)$ and $\psi=f_{0}$.

Genus of $G$ positive. Let $\left(g_{1}, g_{2}\right)$ denote a nonextendible pair of meromorphic functions on $G$. Let $n$ denote the valence of $g_{1}$. Let $P(z, w)$ $=\sum_{0}^{n} A_{k}(z) w^{k}, A_{n}(z)=1$, be the polynomial with coefficients which are rational functions, satisfying $P\left(g_{1}, g_{2}\right)=0$. Relative to the field of rational functions $P$ is irreducible. Since $\left(\phi\left(g_{1}\right), \phi\left(g_{2}\right)\right)$ satisfies $P(z, w)=0$ we conclude that there exists a conformal map $\psi_{0}$ of $F$ into $G$ such that $\phi\left(g_{k}\right)=g_{k} \circ \psi_{0}$ $(k=1,2)$. Given $g \in \mathfrak{M}(G)$, we have $g=\sum_{0}^{n-1}\left(r_{k} \circ g_{1}\right) g_{2}^{k}$ where the $r_{k}$ are rational functions. It follows that $\phi(g)=g \circ \psi_{0}$ and $\psi=\psi_{0}$.

8. We now turn to the proof of Theorem B. Our argument will be based on the study of maps of $\mathfrak{M}(G)$ into the extended plane which share a number of properties with the maps of the form $g \rightarrow g(q)$ where $q$ is a point of $G$. It will be observed that the extended homomorphisms of Royden [4] which are based on valuation theory satisfy the conditions (1), (2), (3) of the following theorem.

THEOREM 8.1. Let $h$ denote a map of $\mathfrak{M}(G)$ into the extended plane. It is assumed that $h$ is continuous if $G$ is not compact. Suppose that $h$ fulfills the following conditions: (1) for each constant $c, h(c)=c$, (2) if $h\left(g_{1}\right)$ and $h\left(g_{2}\right)$ are finite, then $h\left(g_{1}+g_{2}\right)=h\left(g_{1}\right)+h\left(g_{2}\right), h\left(g_{1} g_{2}\right)=h\left(g_{1}\right) h\left(g_{2}\right)$, (3) for $g \neq 0, h(g)=0$ if and only if $h\left(g^{-1}\right)=\infty$. Then there exists a unique $q \in G$ such that $h(g)=g(q)$, $g \in \mathfrak{M}(G)$. 
It is to be observed that (2) and (3) imply: (4) if $h\left(g_{1}\right) \neq 0$ and $h\left(g_{2}\right)=\infty$, then $h\left(g_{1} g_{2}\right)=\infty$.

Proof. $G$ not compact. To be explicit we shall understand that $h$ is continuous in the sense that if $g_{n} \rightarrow g$ uniformly in $G$ (i.e. uniformly on each compact subset of $G)$ then $h\left(g_{n}\right) \rightarrow h(g)$. We start with the observation that, if $g \in \mathfrak{A}(G)$, then $h[\exp (g)] \neq 0, \infty$. Suppose that $h[\exp (g)]=0$. Then $h\left[\exp \left(n^{-1} g\right)\right]=0$ for each positive integer $n$. But $\lim _{n \rightarrow \infty} h\left[\exp \left(n^{-1} g\right)\right]=1$. Contradiction. If $h[\exp (g)]=\infty$, then $h[\exp (-g)]=0$ and again we have a contradiction. Consequently we conclude that $h\left[\sum_{0}^{n} g^{k} / k !\right] \neq \infty$ for $n$ sufficiently large. Hence for $n$ large $h\left(g^{n}\right) \neq \infty$. Hence $h(g) \neq \infty$. If $g \in \mathfrak{A}(G)$ has no zeros then $h(g) \neq 0$.

Let $\Im$ denote the set of $g$ in $\mathfrak{A}(G)$ for which $h(g)=0$. That $\Im$ is an ideal of $\mathfrak{A}(G)$ is immediate. We shall see that $\Im$ is a principal ideal generated by a member of $\mathfrak{A}(G)$ which has precisely one zero, the zero being simple. If $g \in \mathcal{J}$, then $g$ vanishes at some point of $G$.

There exists a function in $\mathfrak{A}(G)$ which vanishes at a given point $q \in G$ and nowhere else on $G$ and has a simple zero at $q$ [2]. We shall denote a generic function of this type by $E_{q}$. We recall (in slightly modified form) the extension of the Weierstrass product theorem to noncompact Riemann surfaces due to H. Florack [2]:

Given a sequence $\left\{q_{k}\right\}$ of distinct points of $G$ which cluster at no point of $G$ and an associated sequence $\left\{n_{k}\right\}$ of positive integers. Then there exist functions $E_{q_{k}}$ such that $\Pi\left(E_{q_{k}}\right)_{n^{n}}$ is a uniformly convergent infinite product in $G$.

We assert that for some $q \in G$ the associated $E_{q} \in \Im$. In fact $\Im$ contains a nonconstant member, say $g_{0}$. If $g_{0}$ has finitely many zeros, say $q_{1}, \cdots, q_{n}$, then $h\left(E_{q_{k}}\right)=0$ for some $k$. If $g_{0}$ has infinitely many zeros, let $\prod\left(E_{q_{k}}\right)^{n_{k}}$ denote a Weierstrass infinite product having the same zeros with the same multiplicities as $g_{0}$. Then $h\left[\Pi\left(E_{q_{k}}\right)^{n_{k}}\right]=0$. Since $\prod_{k>m}\left(E_{q_{k}}\right)^{n_{k} \rightarrow 1}$ uniformly in $G$, we conclude that for some positive integer $m, h\left[\prod_{k \leq m}\left(E_{q_{k}}\right)^{n_{k}}\right]=0$ and hence that $h\left(E_{q_{k}}\right)=0$ for some $k$. Further for only one $q$ do the $E_{q}$ belong to $\Im$. In fact, given $E_{q}, E_{r} \in \Im$, we have $E_{q}-E_{q}(r) \in \Im$ and hence $0=h\left(E_{q}\right)=E_{q}(r)$. It follows that $q=r$. Let $q_{0}$ be the unique $q$ for which $E_{q_{0}} \in \Im$. If $g \in \mathcal{S}, g\left(q_{0}\right)=0$. We conclude that $\Im$ is the principal ideal generated by $E_{q_{0}}$. It follows that $h(g)=g\left(q_{0}\right), g \in \mathfrak{A}(G)$ (cf. [4, Proposition 4]). It is readily established that $h(g)=g\left(q_{0}\right), g \in \mathfrak{M}(G)$.

$G$ compact. Let $g_{1}$ be a nonconstant member of $\mathfrak{M}(G)$. Let $q_{1}, \cdots, q_{m}$ denote the antecedents of $h\left(g_{1}\right)$ with respect to $g_{1}\left(q_{k} \neq q_{l}\right.$ for $\left.k \neq l\right)$. Let $g_{2} \in \mathfrak{M}(G)$ be such that $h\left(g_{2}\right) \neq \infty$, that $g_{2}$ is unramified over 1 and that $g_{2}\left(q_{k}\right)=1(k=1, \cdots, m)$. Such a choice of $g_{2}$ is possible. We have

$$
g_{2}^{n}+\sum_{0}^{n-1}\left(A_{k} \circ g_{1}\right) g_{2}^{k}=0
$$

where $n$ is the valence of $g_{1}$ and the $A_{k}$ are rational functions each of which is finite at $h\left(g_{1}\right)$. We conclude that $h\left(g_{2}\right)=1$. Let $g_{3} \in \mathfrak{M}(G)$ be such that $g_{3}$ 
attains distinct finite values at the antecedents of 1 with respect to $g_{2}$ and that $h\left(g_{3}\right) \neq \infty$. We conclude that $h\left(g_{3}\right)=g_{3}(q)$ for precisely one point $q$ at which $g_{2}$ attains 1 . Now each $g \in \mathfrak{M}(G)$ admits a unique representation of the form

$$
\sum_{0}^{\mu-1}\left(B_{k} \circ g_{2}\right) g_{3}^{k}
$$

where the $B_{k}$ are rational functions and $\mu$ is the valence of $g_{2}$. If $g \in \mathfrak{M}(G)$ is finite at each of the antecedents of 1 with respect to $g_{2}$, then we conclude from the above representation of $g$ that $h(g)=g(q)$. If $g$ is infinite at some antecedent of 1 with respect to $g_{2}$, then for a suitably chosen constant $c$, $(g-c)^{-1}$ is finite at each of the antecedents of 1 with respect to $g_{2}$, and from $h\left[(g-c)^{-1}\right]=[g(q)-c]^{-1}$ we conclude $h(g)=g(q)$.

9. The extended homomorphisms of Royden are defined as follows. Let $K$ denote a field containing the complex constants. A map $\pi$ of $K$ into the extended plane is termed an extended homomorphism provided that there exists a valuation $v$ of $F$ such that $O=\{f \mid \pi(f) \neq \infty\}$ is the valuation ring of $v$, $\{f \mid \pi(f)=0\}$ is the valuation ideal of $v$ and the restriction of $\pi$ to $O$ is a homomorphism preserving the complex numbers. It is readily verified as remarked above that $\pi$ satisfies (1), (2), (3) of Theorem 8.1.

Royden raises the question [4] whether for $\mathfrak{M}(G)$ or subfields, $\pi(g)=g(q)$ for some $q \in G$; in particular the question is raised for the subfield of functions of bounded characteristic. This need not be the case when $G=[0<|z|<1]$. In fact the functions of bounded characteristic in $G$ are precisely the restriction to $G$ of the functions of bounded characteristic in $[|z|<1]$. Let it be agreed that with each function of bounded characteristic in $G$ there is associated the value of its extension to $(|z|<1)$ at 0 . The map so defined is an admissible $\pi$. It cannot be realized by a map of the form $g \rightarrow g(\zeta), 0<|\zeta|<1$.

10. Theorem $B$ is an immediate consequence of Theorem 8.1 and Theorem A. In fact let $p_{0} \in F$ and consider $h(g)=\phi(g)\left(p_{0}\right)$. By Theorem 8.1, there exists $q_{0} \in G$ such that $\phi(g)\left(p_{0}\right)=g\left(q_{0}\right)$. The set $\psi$ of Theorem A is not empty and Theorem B follows. In case $G$ is compact the continuity of $\phi$ is not used in Theorem 8.1 and we have a second proof of Theorem B for $G$ compact with the condition of continuity for $\phi$ omitted.

11. For (continuous) isomorphisms $\phi$ of $\mathfrak{M}(G)$ into $\mathfrak{M}(F)$ which carry complex constants into their conjugates Theorems $\mathrm{A}$ and $\mathrm{B}$ remain valid with obvious modifications. We have $\overline{\phi(g)}=g \circ \psi$ where $\psi$ is an anticonformal map of $F$ into $G$.

12. For the remainder of this paper we shall be concerned with the subfields of $\mathfrak{M}(F)$ which appear as the image of an $\mathfrak{M}(G)$ under the map $\phi_{\psi}$ : $g \rightarrow g \circ \psi$ where $\psi$ is a conformal map of $F$ into $G$. We shall denote by $K_{\psi}$ the image of $\mathfrak{M}(G)$ induced by $\psi$. In particular, we seek to relate $K_{\psi}$ with the mapping properties of $\psi$. 
The first question we consider is the following. Under what circumstances is $\phi_{\psi}^{-1}$ continuous? It is easy to see that $\phi_{\psi}^{-1}$ is continuous if $\psi$ maps $F$ onto $G$. To proceed in the opposite direction, suppose that $\phi_{\psi}^{-1}$ is continuous. If $F$ is compact, $\psi$ is onto. Suppose that $F$ is not compact. We wish to show that $\psi$ is onto. Assume that this is not the case. Let $q \in G-\psi(F)$ and let $g \in \mathfrak{M}(G)$ have a pole at $q$ but at no other point of $G$. We conclude that $K_{\psi}$ contains nonconstant analytic functions. Let $\Re$ denote the ring of analytic functions belonging to $K_{\psi}$. If $\left\{f_{n}\right\}$ is a sequence of functions belonging to $R$ which converges uniformly in $F$ (in the sense of the euclidean metric of the finite plane) and if $g_{n}=\phi_{\psi}^{-1}\left(f_{\dot{n}}\right)$, then $\left\{g_{n}\right\}$ converges uniformly in $G$ (in the sense of the chordal metric). Take $f_{n}=\phi_{\psi}\left(\sum_{0}^{n} g^{k} / k !\right)$. We conclude that $\sum_{0}^{\infty} g^{k} / k$ ! converges uniformly in $G$ and this is impossible. Hence $\psi$ must be onto.

It is readily concluded that: $\phi_{\psi}^{-1}$ is continuous if and only if $K_{\psi}$ is closed in $\mathfrak{M}(F)$.

13. The special situation where $\psi$ has finite constant valence is of interest. Here we may conclude that $\mathfrak{M}(F)$ is a simple algebraic extension of $K_{\psi}$ of finite degree, the degree being the valence of $\psi$. To see this, let $n$ denote the valence of $\psi$ and let $p_{1}, \cdots, p_{n}$ denote $n$ distinct points of $F$ having a common image with respect to $\psi$ and let $f_{0} \in \mathfrak{M}(F)$ take distinct values at the $p_{k}$. Then $f_{0}$ satisfies an equation of the form

$$
f_{0}^{n}+\sum_{0}^{n-1} a_{k} f_{0}^{k}=0
$$

where $a_{k} \in K_{\psi}$ and the polynomial $w^{n}+\sum_{0}^{n-1} a_{k} w^{k}$ is irreducible relative to $K_{\psi}$. Further each $f \in \mathfrak{M}(F)$ admits a unique representation of the form

$$
f=\sum_{0}^{n-1} b_{k} f_{0}^{k}
$$

In the opposite direction we may infer that $\psi$ has a constant finite valence from a hypothesis on $K_{\psi}$ which is considerably weaker than the hypothesis that $\mathfrak{M}(F)$ is a simple algebraic extension of $K_{\psi}$. It suffices to assume that $\mathfrak{M}(F)$ is algebraic over $K_{\psi}$.

We note that, if there exists a sequence $\left\{p_{k}\right\}_{1}^{\infty}$ of points of $F$ tending to the ideal boundary and satisfying $\psi\left(p_{k}\right) \rightarrow q \in G$ and $\psi\left(p_{k}\right) \neq q$, there would exist as a consequence of the Florack extension of the Weierstrass and Mittag-Leffler theorems [2] a function $f \in \mathfrak{A}(F)$ such that $\left\{f\left(p_{k}\right)\right\}$ is dense in the plane. Clearly $f$ cannot be algebraic over $K_{\psi}$. It follows that, if $\mathfrak{M}(F)$ is algebraic over $K_{\psi}, \psi$ has constant finite valence on $G$.

14. Another question relating mapping structure and associated subfield is the following: Let $\psi_{j}$ denote a conformal map of $F$ into $G_{j}(j=1,2)$. Under what circumstances is $K_{\psi_{1}} \subset K_{\psi_{2}}$ ? The answer is very simple: $A$ necessary and sufficient condition that $K_{\psi_{1}} \subset K_{\psi_{2}}$ is that there exist a conformal map $\theta$ of $G_{2}$ into $G_{1}$ such that $\psi_{1}=\theta \circ \psi_{2}$. 
If such a $\theta$ exists, then clearly $g \circ \psi_{1}=(g \circ \theta) \circ \psi_{2} \in K_{\psi_{2}}, g \in \mathfrak{M}\left(G_{1}\right)$. Hence $K_{\psi_{1}} \subset K_{\psi_{2}}$. To establish the theorem in the other direction, for each $g \in \mathfrak{M}\left(G_{1}\right)$ let $\tilde{g}$ denote the member of $\mathfrak{M}\left(G_{2}\right)$ satisfying $g \circ \psi_{1}=\tilde{g} \circ \psi_{2}$. By Theorem A, there exists a conformal map $\theta$ of $G_{2}$ into $G_{1}$ such that $g \circ \theta=\tilde{g}, g \in \mathfrak{M}\left(G_{1}\right)$. From $g \circ \psi_{1}=g \circ\left(\theta \circ \psi_{2}\right)$ and the fact that $\mathfrak{M}\left(G_{1}\right)$ separates $G_{1}$, we have $\psi_{1}=\theta \circ \psi_{2}$.

It is now readily verified that $K_{\psi_{1}}=K_{\psi_{2}}$ if and only if there exists a univalent conformal map $\theta$ of $G_{2}$ onto $G_{1}$ such that $\psi_{1}=\theta \circ \psi_{2}$.

It is to be observed that the mapping $g \rightarrow \tilde{g}$ in the inclusion theorem just established carries the analytic members of $\mathfrak{M}\left(G_{1}\right)$ into analytic members of $\mathfrak{M}\left(G_{2}\right)$. In case $G_{1}$ is not compact, if we were assured a priori of this fact, it would be possible to invoke Royden's theorem [4]. However it is not apparent to me how one can be assured of this fact without the intervention of an argument of the above type since the isomorphism induced by $\psi_{2}$ may carry members of $\mathfrak{M}\left(G_{2}\right)$ with poles into members of $K_{\psi_{2}}$ which are analytic.

15. If we are given that $K_{\psi}$ admits a continuous isomorphism $\phi$ onto $\mathfrak{M}(H)$ where $H$ is a Riemann surface, we may conclude that $\phi^{-1}$ is continuous and that $\psi$ maps $F$ onto $G$. In fact, $\phi \circ \phi_{\psi}$ is a continuous isomorphism of $\mathfrak{M}(G)$ onto $\mathfrak{M}(H)$. Hence $\phi[g \circ \psi]=g \circ \theta$ where $\theta$ is a univalent conformal map of $H$ onto $G$. Hence $(g \circ \theta) \circ\left(\theta^{-1} \circ \psi\right)=\phi^{-1}[g \circ \theta]$. We infer that $\phi^{-1}$ is continuous. Since $\theta^{-1} \circ \psi$ maps $F$ onto $H, \psi$ maps $F$ onto $G$.

16. Continuous automorphisms of $K_{\psi}$. Suppose that $\alpha$ is a continuous automorphism of $K_{\psi}$ which preserves the complex constants. By Theorem B $\alpha[g \circ \psi]=g \circ \psi_{1}, g \in \mathfrak{M}(G)$, where $\psi_{1}$ is a conformal map of $F$ into $G$. Let $\tilde{g}$ denote the unique member of $\mathfrak{M}(G)$ satisfying $\tilde{g} \circ \psi=g \circ \psi_{1}$. By Theorem A, there exists a univalent conformal map $\theta$ of $G$ onto itself such that $\tilde{g}=g \circ \theta$. We have $\theta \circ \psi=\psi_{1}$. Now $\theta$ is not an altogether unrestricted univalent conformal map of $G$ onto itself. Indeed we have: $\theta[\psi(F)] \subset \psi(F)$. If this were not the case, there would exist $q$ in $\theta[\psi(F)]$ but not in $\psi(F)$. Let $g$ denote a function in $\mathfrak{M}(G)$ having a pole at $q$ but analytic everywhere else on $G$. Let $f_{n}=n^{-1} g \circ \psi, n=1,2, \cdots$. Then $f_{n} \rightarrow 0$ uniformly in $F$. But for each $n, \alpha\left(f_{n}\right)$ has a pole at each antecedent of $q$ with respect to $\theta \circ \psi$. The continuity of $\alpha$ is violated.

On the other hand, if $\theta$ is a univalent conformal map of $G$ onto itself which maps $\psi(F)$ into itself, then $g \circ \psi \rightarrow g \circ \theta \circ \psi$ defines a continuous automorphism of $K_{\psi}$ onto itself. The verification of the details is straightforward.

It may happen that $\alpha$ is continuous but that $\alpha^{-1}$ is not. We note that $\alpha\left[g \circ \theta^{-1} \circ \psi\right]=g \circ \psi$. Hence $\alpha^{-1}[g \circ \psi]=g \circ \theta^{-1} \circ \psi$. Hence $\alpha^{-1}$ is continuous also if and only if $\theta^{-1}[\psi(F)] \subset \psi(F)$ or equivalently $\psi(F)=\theta[\psi(F)]$.

An example of an automorphism $\alpha$ whose inverse is not continuous is afforded by: $F=(|z|<1), G=$ extended plane, $\psi(z) \equiv z, \theta(z) \equiv z / 2$.

Several remarks are in order. If $F$ is compact, then every automorphism of $K_{\downarrow}$ preserving the complex constants is continuous. If $G$ is compact, then an arbitrary automorphism $\alpha$ of $K_{\psi}$ onto itself preserving the complex constants 
admits a unique representation of the form $g \circ \psi \rightarrow g \circ \theta \circ \psi$ where $\theta$ is a univalent conformal map of $G$ onto itself and conversely $g \circ \psi \rightarrow g \circ \theta \circ \psi$ is indeed an automorphism of $K_{\psi}$ onto itself preserving the complex constants for each univalent conformal map $\theta$ of $G$ onto itself.

17. We terminate with the statement of two problems which arise naturally in the present setting. (a) Given a subfield $K$ of $\mathfrak{M}(F)$. To determine a necessary and sufficient condition that $K$ be the image of some $\mathfrak{M}(G)$ with respect to a (continuous) isomorphism. (b) What is the status of a separating subfield of an $\mathfrak{M}(F)$ ? In particular, is it dense in $\mathfrak{M}(F)$ as is the case for the field of rational functions in a plane region?

\section{BIBLIOGRAPHY} 315.

1. L. Bers, On rings of analytic functions, Bull. Amer. Math. Soc. vol. 54 (1948) pp. 311-

2. H. Florack, Reguläre und meromorphe Funktionen auf nicht geschlossenen Riemannschen Flächen, Schr. Math. Inst. Univ. Münster, no. 1, 1948, 34 pp.

3. S. Kakutani, Rings of analytic functions, Lectures on Functions of a Complex Variable, University of Michigan, 1955, pp. 71-83.

4. H. L. Royden, Rings of analytic and meromorphic functions, Trans. Amer. Math. Soc. vol. 83 (1956) pp. 269-276.

5. W. Rudin, An algebraic characterization of conformal equivalence, Bull. Amer. Math. Soc. Abstract 61-6-748.

BROWN UNIVERSITY AND

INSTITUTE FOR ADVANCED STUDY 